100 years to pass before solving the mysteries of childhood arthritis and it is to be hoped that a flexible, biologically relevant classification will assist that process.

Department of Rheumatology, University of Birmingham,

T R SOUTHWOOD Birmingham B15 2TT

1 Still GF. On a form of chronic joint disease in children. Medico-Chirurgical Trans 1897;80:47, reprinted in Am J Dis Child 1978;132:195-200.

2 Still GF. Common disorders and diseases of childhood, 4th ed. Oxford: Oxford Medical Publications, 1923.

3 Southwood TR, Woo P. Childhood arthritis: the name game. Br J Rheumatol 1993;32:421-3.

4 Huppertz HI. Viewpoint: time for change in paediatric rheumatology. Rheum Int 1993;13:37-8.

5 Laxer RM. What's in a name: the nomenclature of juvenile arthritis. J Rheumatol 1993;20(s):1-3. 6 Fink C, Task Force for Classification Criteria. A proposal for the development of classification criteria

7 Miller JJ. Proposal for classification criteria for idiopathic arthritides of childhood. J Rheumatol 1995;23:944.

8 Hochberg MC. Classification criteria for childhood arthritic diseases. J Rheumatol 1995;22:1445-6.

9 Giannini EH, Lovell DJ. Interphysician reliability estimates for conventional disease activity measures in JRA. Arthritis Rheum 1994;37(s):S328, 1004.

10 Guzman J, Burgos-Vargas R, Duarte C, Gomez-Mora P. Reliability of the oint examination in children with rheumatoid arthritis. Arthritis Rheum 1994;37(s):S238, 1006.

11 Malleson PN. Proposal for classification criteria for idiopathic arthritides of childhood. J Rheumatol 1995;23:943.

12 Prieur A-M, Ansell BM, Bardfield R, et al. Is onset type evaluated during the first 3 months of disease satisfactory for defining the sub-groups of juvenile chronic arthritis? Clin Exp Rheumatol 1990;8:321-5.

13 Cassidy JT, Levinson JE, Brewer EJ. The development of classification criteria for children with juvenile rheumatoid arthritis. Bull Rheum Dis 1989; 38:1-7.

\title{
Uniform structured formats for scientific communications- how far should we go?
}

Clear, efficient communication is a central aim of any scientific report. Clarity, however, can readily be lost when, as authors, we employ our individual literary style, omit detail that we but not the reader take "as read", present information in long sections without subheadings, and expand reports with comment that relates more to the general topic than the specifics of the study. Following peer review a common request to authors from editors is firstly, to include more detail in the methods and results sections, and secondly, to remove extraneous information and extrapolation from the discussion. Peer review and revision, however, do not always result in optimal presentation of information. There is often disparity between what a study should report and what is actually published. In the case of randomised controlled trials
(RCT) this presents important problems for inclusion in systematic reviews ${ }^{1}$ and the balanced appraisal of knowledge that may determine clinical practice.

The continuing education of investigators is clearly important if we are to maintain high quality research and communication. One-often underestimated-aspect of this is the experience of peer review. Although primarily established to guide selection for publication, peer review serves an important educational role in directing authors to potential problems and caveats of their study and in suggesting ways of improving presentation and discussion. It is for this reason that the Annals editorial office always sends appropriately submitted reports to peer review, even if the Editor's initial appraisal is that the report is sufficiently flawed that it would not be accepted even after

Table 1 CONSORT checklist for randomised controlled trials

\begin{tabular}{|c|c|c|c|c|}
\hline Heading & Subheading & Descriptor & $\begin{array}{l}\text { Was it } \\
\text { reported? }\end{array}$ & $\begin{array}{l}\text { On what } \\
\text { page No? }\end{array}$ \\
\hline Title & & Identify the study as a randomised trial & & \\
\hline Abstract & & Use a structured format & & \\
\hline Introduction & & $\begin{array}{l}\text { State prospectively defined hypothesis, clinical objectives, and planned subgroup or covariate } \\
\text { analyses }\end{array}$ & & \\
\hline \multirow[t]{12}{*}{ Methods } & Protocol & Describe & & \\
\hline & & Planned study population, together with inclusion/exclusion criteria & & \\
\hline & & Planned interventions and their timing & & \\
\hline & & $\begin{array}{l}\text { Primary and secondary outcome measure(s) and the minimum important differences(s), } \\
\text { and indicate how the target sample size was projected }\end{array}$ & & \\
\hline & & $\begin{array}{l}\text { Rationale and methods for statistical analyses, detailing main comparative analyses and } \\
\text { whether they were completed on an intention-to-treat basis }\end{array}$ & & \\
\hline & & Prospectively defined stopping rules (if warranted) & & \\
\hline & Assignment & Describe & & \\
\hline & & Unit of randomisation (eg, individual, cluster, geographic) & & \\
\hline & & Method used to generate the allocation schedule & & \\
\hline & & Method of allocation concealment and timing of assignment & & \\
\hline & & Method to separate the generator from the executor of assignment & & \\
\hline & $\begin{array}{l}\text { Masking } \\
\text { (blinding) }\end{array}$ & $\begin{array}{l}\text { Describe mechanism (eg, capsules, tablets); similarity of treatment characteristics (eg, } \\
\text { appearance, taste); allocation schedule control (location of code during trial and when } \\
\text { broken); and evidence for successful blinding among participants, person doing intervention, } \\
\text { outsome assessors, and data analysts. }\end{array}$ & & \\
\hline \multirow[t]{6}{*}{ Results } & $\begin{array}{l}\text { Participant flow } \\
\text { and follow up }\end{array}$ & $\begin{array}{l}\text { Provide a trial profile (figure) summarising participant flow, numbers, and timing of } \\
\text { randomisationassignment, interventions, and measurements for each randomised group }\end{array}$ & & \\
\hline & Analysis & $\begin{array}{l}\text { State estimated effect of intervention on primary and secondary outcome measures, including a } \\
\text { point estimate and measure of precision (confidence interval) }\end{array}$ & & \\
\hline & & State results in absolute numbers when feasible (eg, $10 / 20$, not $50 \%$ ) & & \\
\hline & & $\begin{array}{l}\text { Present summary data and appropriate descriptive and inferential statisitics in sufficient detail to } \\
\text { permit alternative analyses and replication }\end{array}$ & & \\
\hline & & Describe prognostic variables by treatment group and any attempt to adjust for them & & \\
\hline & & Describe protocol deviations from the study as planned, together with the reasons & & \\
\hline \multirow[t]{2}{*}{ Comment } & & $\begin{array}{l}\text { State specific interpretation of study findings, including sources of bias and imprecision (internal } \\
\text { validity) and discussion of external validity, including appropriate quantitative measures when } \\
\text { possible }\end{array}$ & & \\
\hline & & State general interpretation of the data in light of the totality of the available evidence & & \\
\hline
\end{tabular}




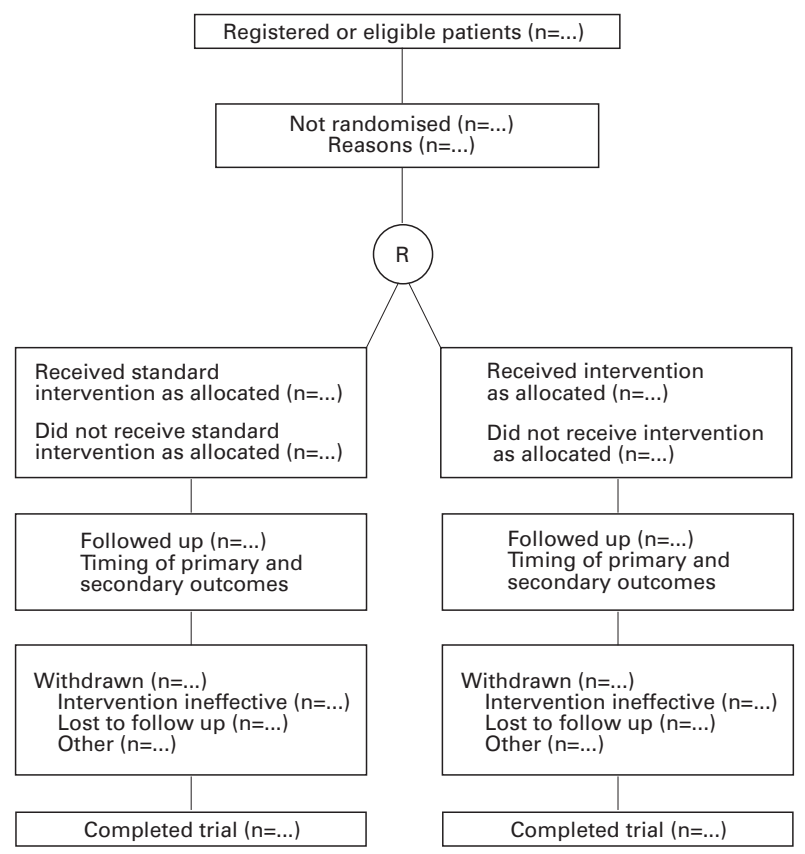

CONSORT flow diagram for randomised controlled trial.

major revision. The authors thus receive more than just a rejection letter from Annals. They obtain a critique of their work and an explanation as to why it was considered unsuitable for publication. Such practice, however, is not always undertaken, especially by larger and busier general medical journals.

An effective means to tailor reports to better fit their purpose is to impose a generic structure on their presentation. The ordered division of a report into headed sections (for example, Summary, Introduction, Results, Methods, Discussion, Acknowledgements, References) facilitates the reader's search for information, but importantly encourages the author to supply information under each heading. In line with many other journals, Annals in 1993 introduced a structured abstract in place of an unstructured summary. This simple manoeuvre means that authors now have to specify the objectives, methods, results, and conclusions of their study, aspects of which are often unclear or omitted from a "summary". With widespread use of computerised literature searches based on abstracts such a descriptive precis is clearly beneficial. From 1997 we have also adopted the CONSORT (Consolidation of Standards for Reporting Trials) format for reporting RCT, ${ }^{2}$ requiring authors to supply a checklist of 21 items (table 1) and a flow diagram showing the progress of subjects through the various stages of the study. The example shown (figure) relates to the most commonly reported two group parallel design $\mathrm{RCT}^{3}$ This strategy to enhance clarity and facilitate the appraisal of RCT will be formally assessed over future years. ${ }^{2}$

Other attempts at standardisation have been supported by a body of international rheumatology journal editors who met first as a group in San Francisco in 1995. An audit by the group in 1996 revealed marked disparity in the referencing format used by rheumatology journals. ${ }^{4}$ The majority of these editors have now agreed to use a single style-the current, fourth edition of the Vancouver
Table 2 A suggested "structured discussion"

Discussion

Conclusions

Summary of principal findings and conclusions

Clinical relevance, if appropriate

Relevant published reports

Placement of study in context of other work

Specific agreements and disagreements with other published work Caveats

Perceived weaknesses of study

Generalisability of data

Future work

Which questions remain unanswered

Direction and nature of required work

system $^{56}$-from January 1997. The following example shows how an original report is referenced in this format:

1 Brown A, Jones B, Smith C, Zahl D. Salicylate intake and subsequent risk of developing knee osteoarthritis. Ann Rheum Dis 1999;58:1984-6.

This agreement by rheumatology journal editors provides a rare example of successful closure of an audit loop in publishing. The group is also developing a common set of rheumatological acronyms and abbreviations and hopes to introduce this by the end of 1997 .

Some, of course, may find uniform requirements and structured formats difficult and even restrictive. Such concerns were raised when the need for more informative structured abstracts was first debated. ${ }^{7}$ In general, however, the benefits of uniform structuring are likely to outweigh the disadvantages expressed in terms of author "freedom" and the difficulty of forcing diverse studies into a common mould. Indeed we might consider going further. For example, it is extremely common for reviewers and editors to request modifications to the discussion section of papers. Subdivision of this section (table 2) may have advantages in reducing overall length and undue extrapolation (a common problem), achieving a better balance of declared strengths and weaknesses of studies (caveats are often underemphasised or omitted by authors), and placing findings in the context of other relevant published work. Other headings could be added, depending on what the function of this section is perceived to be. The views of authors and readers of the Annals on this matter would be welcomed.

MICHAEL DOHERTY
Rheumatology Unit,
City Hospital,
Nottingham
NG5 1PB, UK

1 Moher D, Dulberg CS, Wells GA. Statistical power, sample size, and their reporting in randomized controlled trials. JAMA 1994;272:122-4.

2 Begg CB, Cho M, Eastwood S, Horton R, Moher D, Lokin I, et al. Improving the quality of reporting of randomised controlled trials. The ing the quality of reporting of randomised

3 Fletcher RH, Fletcher SW. Clinical research in general medical journals: a 30-year perspective. N Engl J Med 1979;301:180-3.

4 Doherty M, Arend WP. A uniform reference system for rheumatology journals. Arthritis Rheum (in press).

5 Uniform requirements for manuscripts submitted to biomedical journals. International Committee of Medical Journal Editors. BMJ 1991;302:33841.

6 Uniform requirements for manuscripts submitted to biomedical journals. International Committee of Medical Journal Editors. JAMA 1993 May 5;269:2282-6.

7 Ad Hoc Working Group for Critical Appraisal of the Medical Literature. A proposal for more informative abstracts of clinical studies. Ann Intern Med
1987;106:598-604. 